\title{
Percutaneous Paravalvular Leak Closure
}

\author{
Amar Krishnaswamy, ${ }^{1}$ E Murat Tuzcu² and Samir R Kapadia ${ }^{3}$ \\ 1. Interventional Cardiology Fellowship; 2. Department of Cardiovascular Medicine, Heart and Vascular Institute; \\ 3. Sones Cardiac Catheterization Laboratories, Cleveland Clinic, Ohio, US
}

\begin{abstract}
Symptomatic paravalvular leak (PVL) complicates up to $12 \%$ of surgical valve replacements. When patients present with congestive heart failure and/or haemolysis, reoperation for repeat valve replacement may be undertaken, but presents greater risk and lower likelihood of success than the initial operation. Therefore, percutaneous approaches to PVL closure have been developed by specialists in structural cardiac intervention. Large series demonstrate high levels of procedural success and promising clinical outcomes for this complex intervention. A thorough understanding of multimodality imaging is necessary for the diagnosis of PVL and the safe and successful performance of these closure procedures.
\end{abstract}

\section{Keywords}

Percutaneous paravalvular leak closure, structural cardiac intervention, transoesophageal echocardiography, DynaCT, C-arm computed tomography integration

Disclosure: The author has no conflicts of interest to declare.

Received: 27 January 2014 Accepted: 18 February 2014 Citation: Interventional Cardiology Review, 2013;9(1):44-8

Correspondence: Samir R Kapadia, Heart and Vascular Institute, Cleveland Clinic, 9500 Euclid Avenue, Desk J2-3, Cleveland, OH 44195, US. E: Kapadis@ccf.org

Among patients undergoing surgical valve replacement, $1-5 \%$ of patients with an aortic valve replacement (AVR) and $2-12 \%$ with a mitral valve replacement (MVR) may develop paravalvular regurgitation or 'leak' (PVL). ${ }^{1-3}$ In the era of transcatheter aortic valve replacement (TAVR) with first-generation balloon expandable valves, up to $17 \%$ of patients may be left with moderate or severe PVL, also referred to as paravalvular aortic regurgitation (PAR). ${ }^{4}$ Risk factors for PVL in patients undergoing surgical valve replacement include the use of mechanical valves, severe calcification of the valve annulus, or valve replacement for infectious endocarditis. Similar factors contribute to PVL in the post-TAVR setting, as well as improper pre-procedural valve sizing. ${ }^{5}$

The majority of patients who have symptomatic PVL present with congestive heart failure (CHF) (85\%), and a significant number may have haemolysis (50 \%). ${ }^{6.7}$ Patients who fail medical therapy directed at CHF and/or haemolysis (erythropoietic agents, blood transfusion) should be considered for redo open-heart surgery (OHS) or percutaneous PVL closure. Re-operation must be approached cautiously, as redo surgery usually carries greater risk than a first operation, and recurrence of PVL may be seen in more than one-third of patients who undergo redo OHS for PVL. ${ }^{6}$

As a result, percutaneous PVL closure has recently gained greater favour. First reported in 1992, this procedure has been slowly evolving and is now successfully performed in a number of centres with significant experience in structural cardiac intervention. ${ }^{8-12}$ In this review, we will discuss the imaging diagnosis of PVL and data supporting percutaneous closure, as well as highlight the procedural techniques to accomplish PVL closure.

\section{Diagnosis}

In patients with prior valve replacement, symptoms of $\mathrm{CHF}$ or haemolysis should merit further evaluation for PVL. Transthoracic echocardiography (TTE) is important to establish ventricular function and overall valve assessment. It is important to understand, however, that prosthetic valve shielding may not provide an adequate characterisation of PVL and appropriate diagnosis may require further imaging using transoesophageal echocardiography (TOE). In some rare situations, it may be unclear whether the leak is intra- or paravalvular by both TTE and TOE; intracardiac echocardiography (ICE) may be helpful in these situations.

\section{Mitral Paravalvular Leak}

The 'clock face' nomenclature of the mitral valve (MV) as seen from the left atrium, or 'surgeon's view', facilitates communication among different specialists (see Figure 1). Most PVLS occur anteromedially (10 to $11 \mathrm{O}^{\prime}$ clock position) and posterolaterally (5 to 6 o'clock position) ${ }^{7,10}$

As discussed above, TTE may provide the diagnosis and location of PVL (see Figure 2). However, TOE is usually necessary to define the extent of PVL, and understanding the relationship of TOE angles is imperative to an accurate localisation and subsequent treatment (see Figure 3). Three-dimensional (3D) TOE may be beneficial to localise the PVL, but is sometimes limited by shadowing artifact or echo dropout (see Figure 4).

\section{Aortic Paravalvular Leak}

The clock face of the aortic valve (AV) shares the 12 o'clock position with the MV (see Figure 1). Aortic PVLs are most commonly 
Figure 1: Clock Face Designation of the Mitral and Aortic Valves from the Left Atrial Side

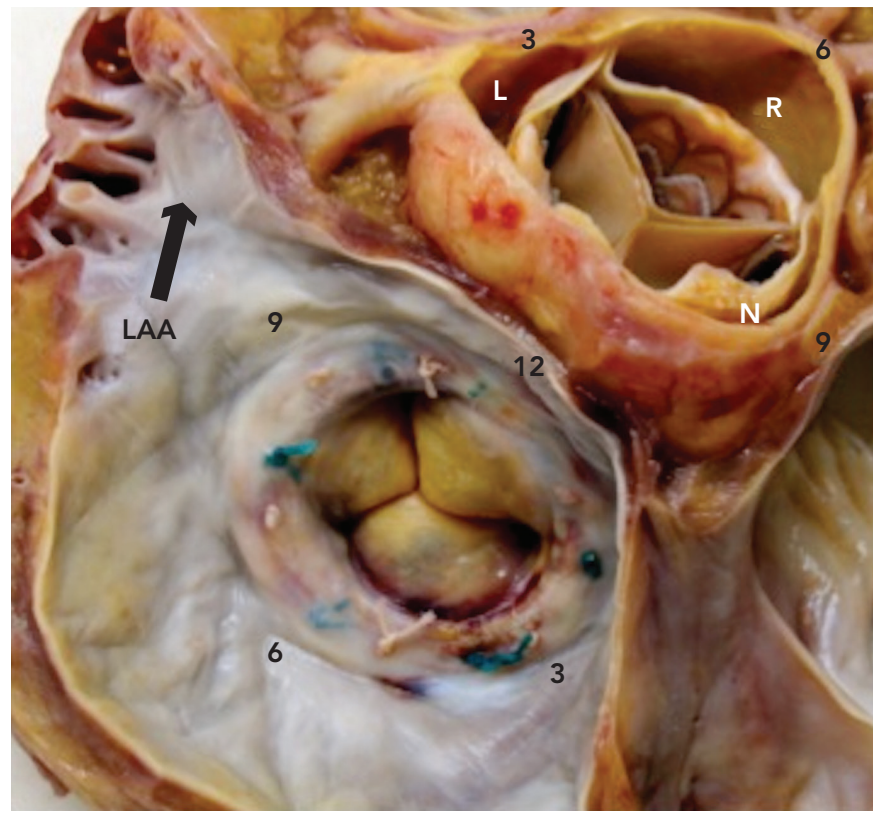

$L=$ left coronary cusp; $L A A=$ left atrial appendage; $N=$ non-coronary cusp; $R=$ right coronary cusp. Reproduced with permission from Krishnaswamy, et al., 2014.14

Figure 2: TTT Localization of Mitral PVL
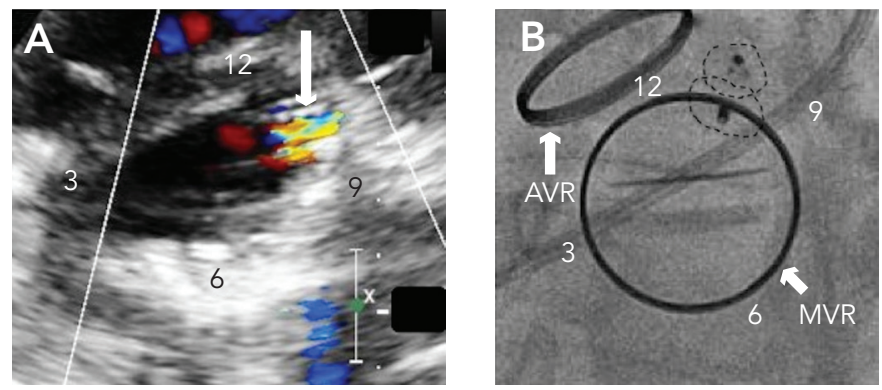

(A) Transthoracic echocardiogram demonstrates mitral paravalvular leak at $10 \mathrm{o}^{\prime} \mathrm{clock}$ in the parasternal short-axis view. (B) Placement of a $6 \mathrm{~mm}$ Amplatzer Vascular Plug Device in the paravalvular leak.

in the paravilic valve replacement; $M V R=$ mitral valve replacement. Reproduced with

permission from Krishnaswamy, et al., 2014.14

encountered at the 7 to 11 o'clock position (46\%), followed by the 11 to 3 o'clock position (36\%). ${ }^{10}$ We find it helpful to also identify the location of the PVL with respect to the native coronary cusp, which more easily translates to the fluoroscopic relationships with which interventionists are familiar. Figure 5 demonstrates the AV in fluoroscopic projection and TOE.

\section{Outcomes of Percutaneous Paravalvular Leak Closure}

While a number of groups have demonstrated successful percutaneous PVL closure in small series' and case reports, Ruiz and colleagues and Sorajja and colleauges have provided the largest published experiences. ${ }^{9}$ Ruiz and colleagues performed 57 PVL procedures in 43 patients, with a procedural success of $86.0 \%$ and a 30-day all-cause mortality rate of $5.4 \% .{ }^{11}$ As a point of reference, surgical series' have demonstrated a mortality of $6 \% .6,10$ Haemolysis was a common finding, seen as the reason for the procedure in $14 \%$ and in combination with CHF among $70 \%$. Despite the fact that $35 \%$ of patients actually developed worsening haemolysis after the procedure, the number
Figure 3: Localisation of Paravalvular Leak Using Transoesophageal Echocardiography

A
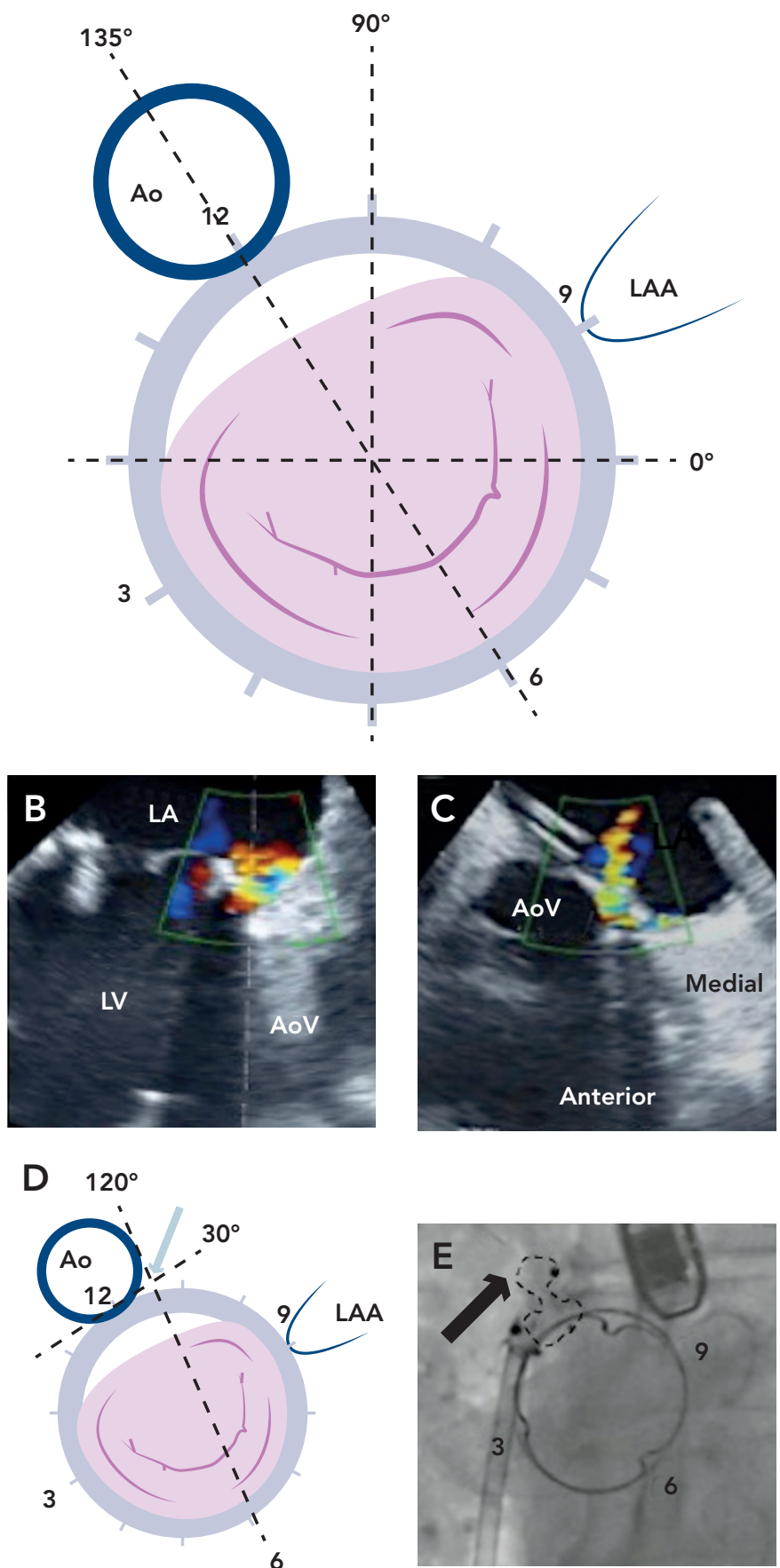

(A) The angles shown refer to the cuts made by a TOE imaging crystal. The numbers correspond to the clock face perspective of the MV (MV is shown from the $L V$; clock face numbering is as viewed from the LA). (B) TOE 120-degree view. (C) TOE 30-degree short-axis view. (D) The TOE planes are shown on the MV clock face (LV view) with the intersection (arrow) defining the PVL origin. (E) Deployment of the Amplatzer muscular VSD occluder (arrow) confirms the initial leak localisation by TOE (LAO projection).

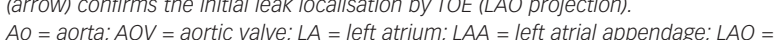
left anterior oblique; $L V=$ left ventricle; $M V=$ mitral valve; $P V L=$ paravalvular leak; TOE $=$ transoesophageal echocardiography; VSD = ventricular septal defect. Adapted with permission from Krishnaswamy, et al., 2014.14

of patients requiring erythropoietic agents of regular transfusion decreased from 56 to $5 \%$. In this series, 10 patients required a redo percutaneous procedure, and two required three procedures total. This not only demonstrates the safety of repeat percutaneous procedures, but the idea that continued valve dehiscence may lead to new or worsening leaks. 
Figure 4: Three-dimensional Transoesophageal Echocardiography Localisation of Mitral Paravalvular Leak Closure
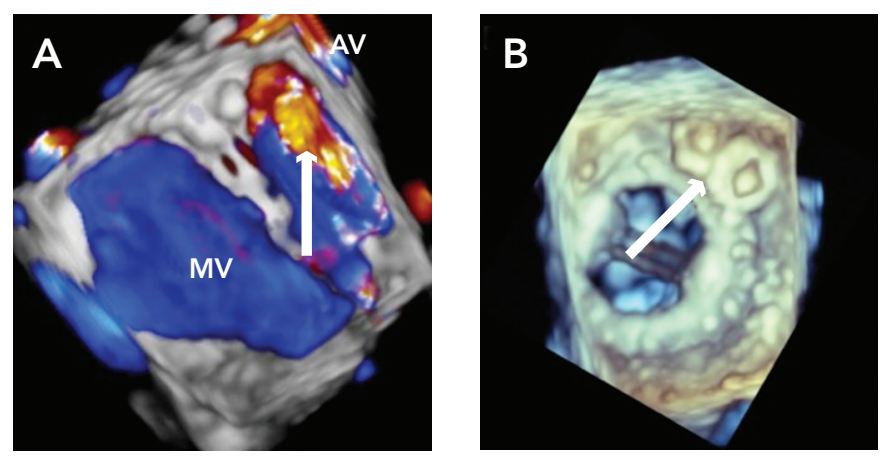

(A) Left atrial view with colour Doppler demonstrates the leak (arrow). (B) Two AVP II plugs in Place (arrow). $A V=$ aortic valve; $A V P=A m p$

with permission from Chenier, et al., 2013.16

Figure 5: Aortic Paravalvular Leak
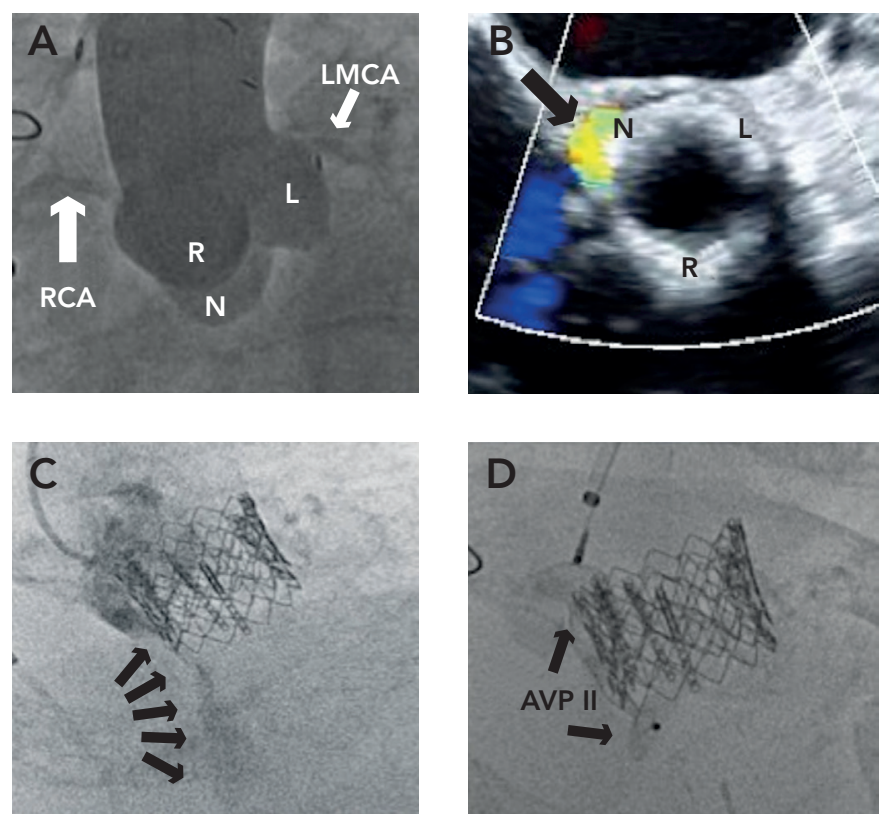

(A) Aortic root angiogram in the LAO projection demonstrates cusp anatomy. (B)

Transoesophageal echocardiogram short-axis view $\left(45^{\circ}\right)$ demonstrates PVL at the junction of right and non-coronary cusps in a patient with prior transcatheter aortic valve replacement. (C) Aortic root angiography in the same patient (LAO projection). (D) Amplatzer vascular plug II deployed in the PVL. AVP = Amplatzer vascular plug; $L=$ left coronary Cusp; $L M C A=$ left main coronary artery; $N=$ non-coronary cusp; $R=$ right coronary cusp; $R C A=$ right coronary artery. Adapted with permission from Krishnaswamy, et al., 2014.14

Sorajja and colleagues published their short-term outcomes of closure for 141 defects (115 patients, $78 \%$ mitral, $22 \%$ aortic) and long-term outcomes on closure of 154 defects (126 patients).11,13 Heart failure outcomes were substantially improved: despite $93 \%$ of patients presenting with $\mathrm{CHF}, 72 \%$ had none or minimal dyspnoea at three-year follow-up. Procedural success was enjoyed by $77.0 \%$ of patients, and $8.7 \%$ experienced a major adverse event at 30-days. Importantly, >3+ PVL was seen in only $10 \%$ of patients post-closure. One patient required emergent surgery due to valve interference by a device that could not be retrieved, there were no procedural deaths, and survival was $64 \%$ at three years.

It is difficult to accurately compare survival in the series of percutaneous PVL closure with those in surgical series. As this is a procedure still in its infancy, with surgery often performed without
Figure 6: Use of Intracardiac Echocardiography to Guide Mitral Paravalvular Leak Closure
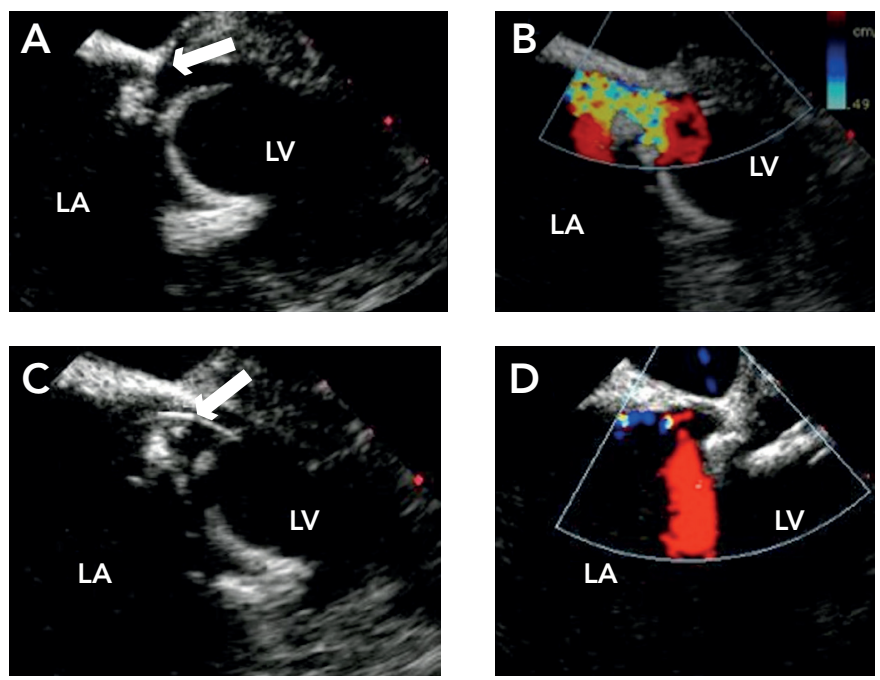

(A) 2D demonstration of the medial PVL; (B) Color Doppler demonstration of the PVL; (C) Wire across the PVL; (D) Minimal residual leak after device closure. Adapted with permission from Krishnaswamy, et al., 2014.14

Figure 7: Mitral Paravalvular Leak Closure Using Computed Tomography Overlay Guidance
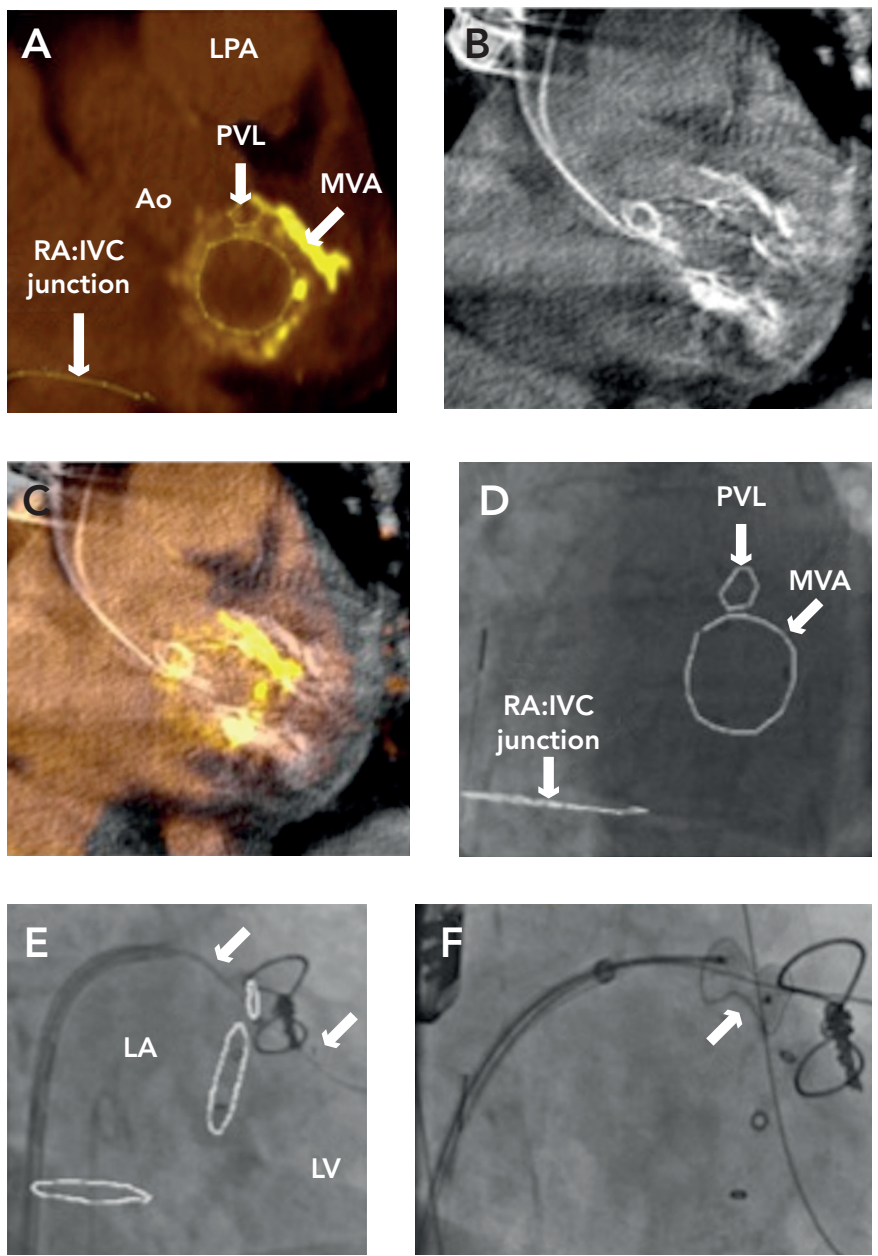

(A) Pre-procedural MDCT marked with points of interest. (B) C-arm CT acquisition (C) MDCT:DynaCT registration. (D) Fluoroscopic overlay of MDCT markings. (E) Wiring the PVL using CT-mark guidance (arrows). (F) Device in place (arrow). $A O=$ aorta; $C T=$ computed tomography; $I V C=$ inferior vena cava; $L A=$ left atrium; $L P A=$ left pulmonary artery; $L V=$ left ventricle; $M V A=$ mitral valve anulus; $P V L=$ paravalvular leak $: R A=$ right atrium.

Reproduced with permission from Krishnaswamy, et al., 2014.15 
consideration of, or access to, percutaneous closure, those patients presenting for percutaneous therapy are often far more co-morbid than their peers who are taken for surgery. Nevertheless, the available data suggest safety of this approach and substantial functional improvement is enjoyed by patients undergoing percutaneous PVL closure. Given the high risks and poor results of reoperation, percutaneous PVL closure presents a promising treatment that is likely to improve with time and innovation.

\section{Procedural Details}

A more thorough description of percutaneous PVL closure techniques can be found elsewhere. ${ }^{14}$ Briefly, percutaneous mitral PVL closure can be performed via femoral vein access and transseptal puncture, left ventricular (LV) apical access, or retrograde via the femoral artery. Closure of aortic PVL is most effectively accomplished retrograde via the femoral artery. Tricuspid PVL can be accomplished via the jugular vein. The choice of access site should be decided on the basis of PVL location, support required for delivery of the bulky closure devices, and presence of other mechanical prostheses that may interfere with wire-snaring/externalisation. As we perform the majority of our PVL closure procedures without general anaesthesia or endotracheal intubation, minimising TOE-probe dwell time is essential for patient comfort. We therefore rely on ICE (see Figure 6) for transseptal puncture and when possible for PVL guidance (see Figure 6). We have also demonstrated previously the feasibility of integrating computed tomography (CT) data onto the realtime fluoroscopic image using a C-arm based CT acquisition (Syngo DynaCT, Siemens Healthcare, Forchheim, Germany) (see Figure 7).15 These techniques allow us to place the TOE probe only after the device is in place to confirm adequate PVL closure.

\section{Choice of Device}

There are no devices created specifically for percutaneous PVL closure; those that are used are created for other applications, such as closure of septal defects or vascular plugs (see Figure 8). An important part of planning the percutaneous PVL closure is understanding the shape and size of the defect. We find that given the crescentic shape of PVLS, and the generally round shape of the devices, multiple devices are often necessary to adequately close the leak. It is important, especially in the setting of mechanical valve replacements, to be watchful of impingement on the prosthetic valve apparatus.

We most often use the AmplatzerTM Vascular Plug II (AVP II; St. Jude Medical, Minnesota, US), which consists of a nitinol cylinder with a nitinol disc on either side. The AVP I is a single cylinder design, making it less stable and effective in our experience, and the AVP III is not available in the US. We find that the use of atrial septal defect (ASD) occlusion devices is often complicated by the large discs that can interfere with the prosthetic valve, and ventricular septal defect (VSD) closure devices are quite stiff and often result in worsening haemolysis. Patent ductus arteriosus (PDA) occluders are available in limited sizes, but are sometimes helpful when the AVP ॥ discs interfere with valve leaflet motion.

\section{Complications of Paravalvular Leak Closure}

Percutaneous PVL closure in trained centres has demonstrated good efficacy and safety. It is important in the performance of these procedures, to be aware of the potential complications in order to plan accordingly.
Figure 8: Devices Used for Paravalvular Leak Closure
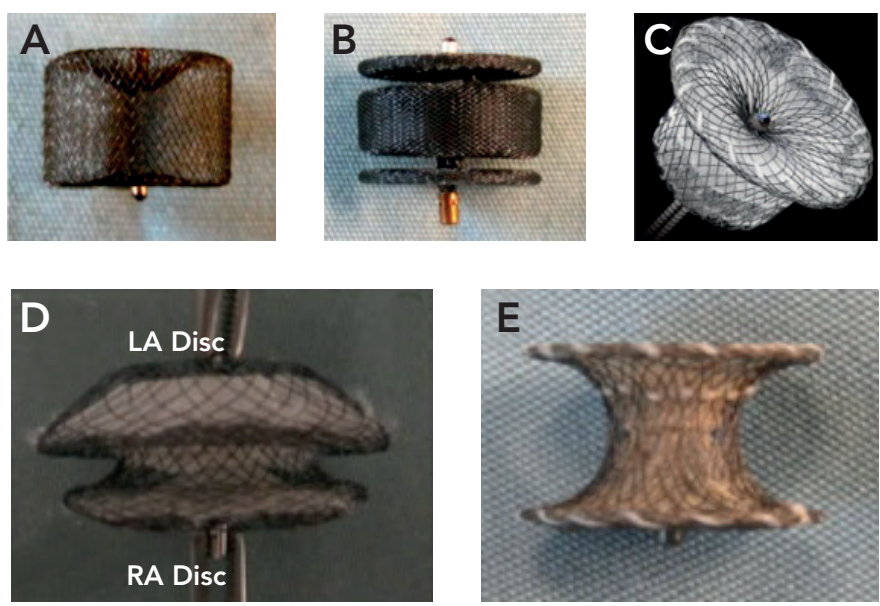

(A) Amplatzer Vascular Plug I (AVP I). (B) Amplatzer Vascular Plug II (AVP II). (C) Amplatzer patent ductus arteriosus (PDA) occluder. (D) Amplatzer atrial septal defect (ASD) occluder. (E) Amplatzer Muscular ventricular septal defect (VSD) Occluder. $L A=$ left atrium; $R A=$ right atrium. Reproduced with permission from Krishnaswamy, et al., 2014.14

The atrioventricular node is situated at the junction of the interatrial septum and the interventricular septum. Therefore, closure of PVLS close to this position can be complicated by complete heart block at the time of device implantation. Pre-emptive temporary pacemaker placement may be reasonable in such cases.

Care must be taken to observe mechanical valve function fluoroscopically and by echocardiography to assure that the PVL closure device does not produce valve dysfunction prior to release. In our experience, it is rare that PVL closure cannot be completed due to valve interference, though attempt at different devices and sizes may be necessary for a successful procedure.

In patients for aortic PVL, proximity to the native right and left coronary arteries should be considered, along with the relative size of the aortic sinus. In some cases, it is helpful to take an aortic root angiogram to further define this space prior to the placement of a PVL closure device.

Embolisation of the occluder devices has been reported in $<1 \%$ to $5 \%$ of large series'. 10,13 A sudden change in symptoms or atrial or ventricular ectopy may be early clues to device embolisation. When it does occur, percutaneous retrieval is usually performed successfully using snare devices and/or bioptomes. Alternatively, if the device is permanently lodged within the LV without significant risk for further mobilisation, consideration can be given to leaving it in place with monitoring by CT or echocardiography during follow-up. ${ }^{10}$ Echocardiographic follow-up is also important to reassess device placement and integrity of the valve itself as further valve dehiscence over time has been reported.

\section{Conclusions}

Patients with cardiac valve replacement may suffer from PVL in the acute, subacute or chronic phases after cardiac surgery. These patients present most commonly with $\mathrm{CHF}$, though a significant number also have debilitating haemolysis. As surgical reoperation carries great risk and chance for PVL recurrence, percutaneous strategies have been developed. These therapies are gaining favour as operators trained in structural cardiac intervention have developed a greater understanding of this procedure and made significant improvements in technique. While no specific trials of percutaneous 


\section{Structural}

versus surgical closure exist, many high-volume interventional and consideration may be given to the percutaneous approach as the centres have been taking care of larger numbers of these patients, first-line treatment strategy in carefully selected patients.

1. Jindani A, Neville EM, Venn G, Williams BT, Paraprosthetic leak: a complication of cardiac valve replacement, I Cardiovasc Surg (Torino), 1991;32:503-8.

2. Hammermeister K, Sethi GK, Henderson WG, et al., Outcomes 15 years after valve replacement with a mechanical vers a bioprosthetic valve: final report of the Veterans Affais randomized trial, I Am Coll Cardiol, 2000;36:1152-8.

3. Ionescu A, Fraser AG, Butchart EG, Prevalence and
clinical significance of incidental paraprosthetic valv regurgitation: a prospective study using transoesophagea echocardiography, Heart, 2003;89:1316-21.

4. Rodes-Cabau J. Transcatheter aortic valve implantation: Current and future approaches, Nature Reviews Cardiology, 2012;9:15-29.

5. Jilaihawi H, Kashif M, Fontana G, et al., Cross-sectional computed tomographic assessment improves accuracy of aortic annular sizing for transcatheter aortic valve eplacement and reduces the incidence of paravalvular aortic regurgitation, J Am Coll Cardiol, 2012;59:1275-86.

6. Genoni M, Franzen D, Vogt P, et al., Paravalvular leakage after mitral valve replacement: Improved long-term survival with aggressive surgery? Eur / Cardiothorac Surg, 2000:17:14-9. De Cicco G, Russo C, Moreo A, et al., Mitral valve

periprosthetic leakage: Anatomical observations in 135 patients from a multicentre study, Eur I Cardiothorac Surg. 2006;30:887-91

8. Hourihan M, Perry SB, Mandell VS, et al., Transcatheter umbrella closure of valvular and paravalvular leaks, I Am Coll Cardiol, 1992,20:1371-7.

9. Kim MS, Casserly IP, Garcia JA, et al., Percutaneous transcatheter closure of prosthetic mitral paravalvular leaks: are we there yet?, JACC Cardiovasc Interv, 2009,2:81-90. 0. Ruiz CE, Jelnin V, Kronzon I, Dudiy Y, et al., Clinical outcomes in patients undergoing percutaneous closure of periprosthetic paravalvular leaks, I Am Coll Cardiol, 2011;58:2210-7.

11. Sorajja P, Cabalka AK, Hagler DJ, Rihal CS, Long-term follow-up of percutaneous repair of paravalvular prosthetic regurgitation, I Am Coll Cardiol, 2011;58:2218-24. repair of paravalvular regurgitation: characteristics and outcomes of 45 patients at cleveland clinic, Presented at: Society for Cardiovascular Angiography and Interventions Scientific Sessions, Orlando, Florida, US, 9 May 2013.

13. Sorajja P, Cabalka AK, Hagler DJ, Rihal CS, Percutaneous repair of paravalvular prosthetic regurgitation: Acute and 30-day outcomes in 115 patients. Circulation, Circ Cardiovasc Intery 2011;:4:314-2

14. Krishnaswamy A, Tuzcu EM, Kapadia SR, Paravalvular leak closure Interventional Procedures for Adult Structural Heart Disease. eds Lasala J \& Rogers J. Elsevier, 2014.

15. Krishnaswamy A, Tuzcu EM, Kapadia SR, Integration of MDC and fluoroscopy using C-arm computed tomography to guide structural cardiac interventions in the cardiac catheterization laboratory, Catheter Cardiovasc Interv, 2014 [Epub ahead of print]

16. Chenier M, Tuzcu EM, Kapadia SR, Krishnaswamy A, Multimodality imaging in the cardiac catheterization laboratory: a new era in sight, Interventional Cardiology, 2013:5:335-44. 